\title{
ANÁLISE DE FERRAMENTAS UTILIZADAS PARA AVALIAR O IMPACTO DA DISFAGIA EM QUALIDADE DE VIDA
}

Maria Paula R D Bezerra ${ }^{1}$, Isadora Soares Lopes ${ }^{1}$, Larissa M Silva ${ }^{1}$, Henrique de Paula Bedaque$^{2}$, Lidiane M B M Ferreira ${ }^{3}$

1. Acadêmicas de Medicina da Universidade Federal do Rio Grande do Norte (UFRN)

2. Residente em Otorrinolaringologia da UFRN

3. Professora Adjunta do Departamento de Cirurgia da UFRN

Study performed at Otorhinolaryngology and Head and Neck Service at Onofre Lopes University Hospital, Brazil.

Financial support: None.

Conflicts of interest: None.

Corresponding author: Otorhinolaryngology and Head and Neck Service at Onofre Lopes University Hospital - Av. Nilo Peçanha, 619A - Petrópolis, Natal - RN CEP: 59012-300.

E-mail: mpaulabezerra26@gmail.com.

Submitted: jun 04; accepted after revision, oct 27, 2021.

\section{RESUMO}

Introdução: Disfagia orofaríngea é um sintoma presente em diversas patologias, que sabidamente leva a um comprometimento na qualidade de vida. Algumas ferramentas, principalmente questionários auto aplicados, estão disponíveis para investigar qualidade de vida nos pacientes com disfagia. Entretanto, eles diferem em vários aspectos, como os domínios avaliados, número de itens, opções de resposta e sistemas de pontuação. A presente revisão sistemática tem como objetivo avaliar as ferramentas disponíveis para análise e quantificação objetiva do impacto na qualidade de vida de pacientes com desordens da deglutição, e quais estão devidamente validadas com esse propósito. Metodologia: Foi realizada uma busca sistemática nas bases de dados MEDLINE (EBSCO), SciELO e PubMed, que incluiu os descritores: "deglutition disorders" AND "quality adjusted life years" OR "sickness impact profile" OR "indicators of quality of life", englobando os artigos publicados nos últimos 10 anos. Os critérios de inclusão foram: 1) abordar disfagia orofaríngea em humanos; 2) correlacionar essa condição clínica com seu impacto na qualidade de vida; 3) avaliar qualidade de vida através de instrumentos objetivos. Em cada estudo, avaliou-se: $O$ desenho, a população-alvo do instrumento desenvolvido, a validação dos questionários, a conclusão do estudo e a aplicabilidade real da ferramenta avaliada. Resultados: Um total de 335 artigos foram encontrados com essa estratégia de busca. Após exclusão dos duplicados, 310 evidências foram analisadas pelo título e resumo, e destas 295 foram excluídas imediatamente por não preencherem os critérios de inclusão. Quinze artigos foram lidos 
Análise de ferramentas utilizadas para avaliar o impacto da disfagia em qualidade de vida Bezerra MPRD, et al

na íntegra, dos quais dez foram incluídos na análise qualitativa. Conclusão: Diversas ferramentas são utilizadas para avaliar a qualidade de vida relacionada à saúde em pacientes com disfagia orofaríngea, mas a maioria não está devidamente validada, ou seu uso é sustentado por estudos de baixa qualidade metodológica. O SWAL-QOL é a ferramenta mais estudada, traduzida para mais línguas e cujas análises psicométricas mostraram melhores resultados, mas novos estudos vêm questionando a qualidade desse instrumento. Assim, reforçamos a importância de estudar objetivamente a correlação entre qualidade de vida de disfagia orofaríngea, mas reiteramos a necessidade de melhores estudos para desenvolvimento de ferramentas com melhor acurácia.

Descritores: Disfagia orofaríngea; Qualidade de vida; Questionários.

\section{INTRODUÇÃO}

A disfagia é uma condição clínica debilitante caracterizada pela disfunção no mecanismo de deglutição ou inabilidade de transferir de forma segura e eficiente o bolo alimentar da boca ao estômago ${ }^{1}$. É um sintoma comum, presente em $16 \%$ da população geral em algum momento da vida, e pode ser consequência de diversas comorbidades, como sequelas de acidente vascular encefálico e câncer de cabeça e pescoço. Sua presença está associada a um aumento de morbidade e mortalidade dos pacientes afetados $^{2}$.

Além disso, sabe-se que os problemas na deglutição trazem complicações graves, como má nutrição e desidratação ${ }^{3}$, além de engasgos e aspiração traqueobrônquica de conteúdo alimentar, os quais contribuem para a ocorrência de pneumonias aspirativas ${ }^{4}$. Mais do que isso, a disfagia agrega um severo impacto psicossocial e está associada à redução na qualidade de vida. A saber, cerca de $41 \%$ dos pacientes com disfagia referem ansiedade ou pânico no horário das refeições, 36\% evitam comer em público devido ao problema para deglutir e menos da metade referem prazer no momento da alimentação. Além de reduzir a interação social e levar a sérios problemas psíquicos ${ }^{5}$.

Assim, considerando o impacto da disfagia na qualidade de vida relacionada à saúde e compreendendo o impacto dos sintomas e comorbidades na gravidade deste problema, avaliar objetivamente esse parâmetro permite entender o impacto de terapêuticas empregadas ${ }^{6}$. Algumas ferramentas estão disponíveis para investigar qualidade de vida nesses pacientes, em sua maioria questionários auto aplicados e, alguns deles, voltados a patologias específicas. Embora o objetivo comum seja acessar a qualidade de vida dos pacientes com disfagia, eles diferem em termos dos domínios avaliados, número de itens, opções de resposta e sistemas de pontuação ${ }^{7}$.

Uma ferramenta de avaliação adequada deve ser validada e ter parâmetros que permitam avaliar sua sensibilidade e confiabilidade, além de apresentar boa capacidade discriminatória, congruência cultural e linguística. Um questionário que não fornece boa 
Análise de ferramentas utilizadas para avaliar o impacto da disfagia em qualidade de vida Bezerra MPRD, et al

diferenciação entre os grupos - sintomático grave e leve, por exemplo - tem pouca utilidade clínica ${ }^{8-9}$.

A presente revisão sistemática tem como objetivo avaliar as ferramentas disponíveis para análise e quantificação objetiva do impacto na qualidade de vida de pacientes com desordens da deglutição, e quais estão devidamente validadas com esse propósito.

\section{METODOS}

Em outubro de 2020, foi realizada uma busca sistemática na literatura nas bases de dados MEDLINE (EBSCO), SciELO e PubMed, que incluiu os seguintes descritores em ciências da saúde: "deglutition disorders" AND "quality adjusted life years" OR "sickness impact profile" OR "indicators of quality of life". Foram pesquisados artigos publicados nos últimos dez anos, em inglês, espanhol, português e francês. Artigos duplicados, encontrados em bases diferentes ou em idiomas diferentes, foram excluídos.

A seleção dos artigos elegíveis deu-se pela leitura de título e resumo. Cada artigo foi avaliado para elegibilidade por duas pesquisadoras de forma independente, e as discordâncias foram resolvidas por uma terceira pesquisadora, que decidia a favor ou contra a inclusão do trabalho na revisão. Certas divergências foram discutidas em conjunto. Os critérios de inclusão foram: 1) abordar disfagia orofaríngea em humanos; 2) correlacionar essa condição clínica com seu impacto na qualidade de vida; 3) avaliar qualidade de vida através de instrumentos objetivos - podendo incluir estudos de validação de ferramentas. Foram excluídos estudos de eficácia terapêutica que usavam melhoria na qualidade de vida como desfecho comparativo entre duas intervenções distintas, ou cuja análise de melhoria na qualidade de vida fora dado secundário. Todos os desenhos de estudo com as informações buscadas nessa revisão foram incluídos.

Em relação aos dados coletados de cada estudo: avaliou-se se o desenho do estudo era apropriado, qual a população-alvo do instrumento desenvolvido, se os questionários elaborados foram validados em uma segunda amostra populacional (validade externa), qual a conclusão retirada do estudo e a aplicabilidade real da ferramenta avaliada. 
Análise de ferramentas utilizadas para avaliar o impacto da disfagia em qualidade de vida Bezerra MPRD, et al

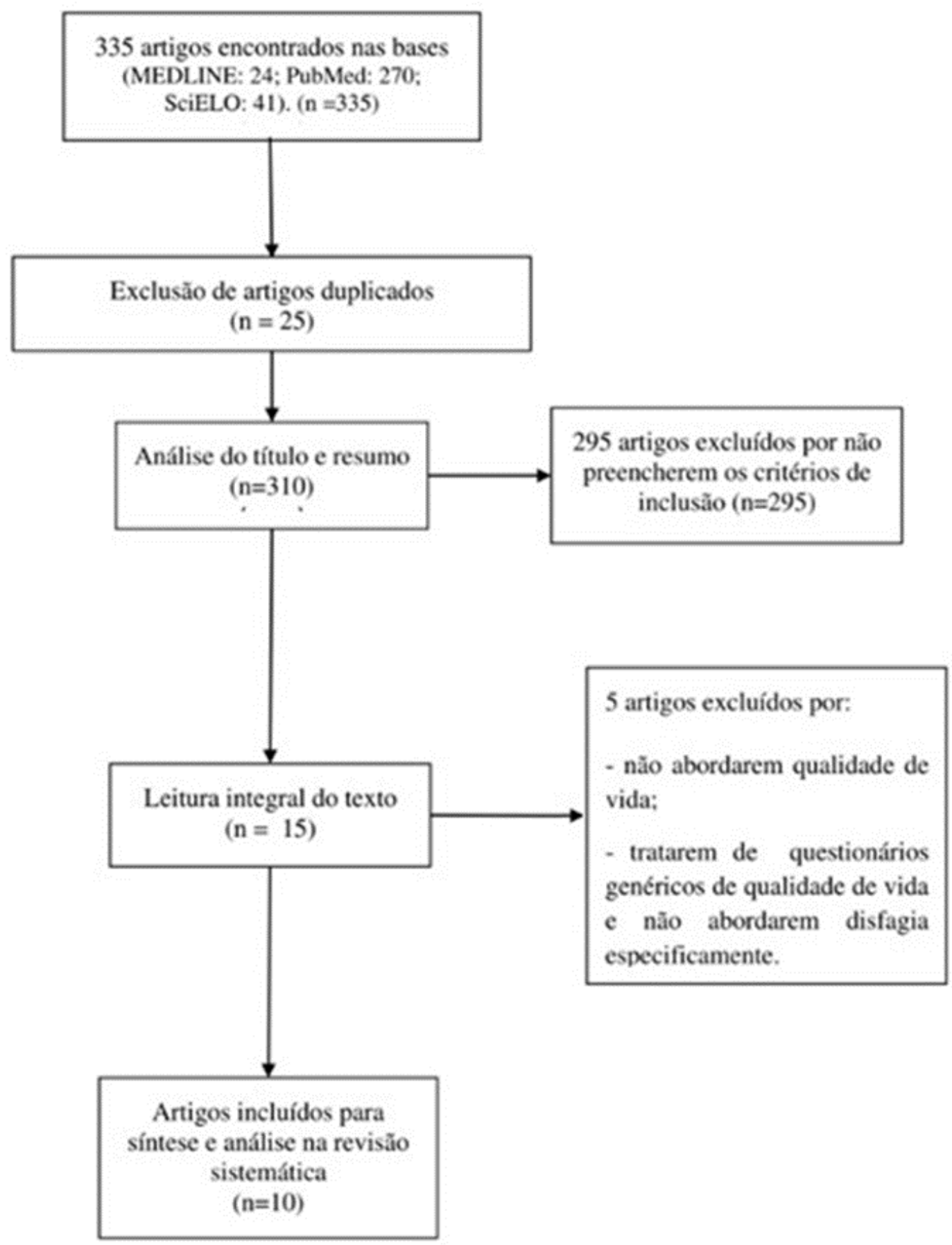

Figura 1 - Fluxograma de seleção dos estudos

\section{RESULTADOS}

Um total de 335 artigos foram encontrados com essa estratégia de busca (MEDLINE: 24; PubMed: 270; SciELO: 41). Após exclusão dos duplicados, 310 evidências foram analisadas pelo título e resumo, e destas 295 foram excluídas imediatamente por não preencherem os critérios de inclusão. Quinze artigos foram lidos na íntegra, dos quais dez foram incluídos na análise qualitativa e estão descritos na tabela 1. 
Tabela 01 - Descrição qualitativa dos artigos científicos incluídos na revisão, com nome do artigo, autor principal, questionário avaliado e resultados obtidos.

\begin{tabular}{|c|c|c|c|}
\hline Artigo & $\begin{array}{l}\text { Autor principal } \\
\text { (Ano) }\end{array}$ & Questionário avaliado & Resultados \\
\hline $\begin{array}{l}\text { Using Rasch Analysis to Evaluate the } \\
\text { Reliability and Validity of the } \\
\text { Swallowing Quality of Life } \\
\text { Questionnaire: An Item Response } \\
\text { Theory Approach10 }\end{array}$ & $\begin{array}{l}\text { Cordier, R. } \\
\text { (2018) }\end{array}$ & $\begin{array}{l}\text { O Swallowing Quality of Life questionnaire } \\
\text { (SWAL-QOL) - Tradução livre, Questionário } \\
\text { de Qualidade de Vida em Deglutição }\end{array}$ & $\begin{array}{l}\text { Os achados do estudo não defendem o uso da versão atual do } \\
\text { SWAL-QOL. Através da análise Rasch, esse instrumento não é } \\
\text { capaz de fornecer boa capacidade discriminatória e apresenta } \\
\text { falhas e limites imprecisos para discernimento das categorias. } \\
\text { Certos itens estão inadequados. Recomenda-se uma } \\
\text { reelaboração do questionário. }\end{array}$ \\
\hline $\begin{array}{l}\text { Relationship between swallow- } \\
\text { specific quality of life and fiber-optic } \\
\text { endoscopic evaluation of } \\
\text { swallowing findings in patients with } \\
\text { head and neck cancer11 }\end{array}$ & $\begin{array}{l}\text { Florie, M. } \\
\text { (2016) }\end{array}$ & $\begin{array}{l}\text { MD Anderson Dysphagia Inventory } \\
\text { (MDADI) - Tradução livre, Inventário de } \\
\text { Disfagia MD Anderson. }\end{array}$ & $\begin{array}{l}\text { Esse estudo analisou pacientes com disfagia e diagnóstico de CA } \\
\text { de cabeça e pescoço ( } n=63 \text {, FOIS: } 5-6 \text { ). De modo geral, não existe } \\
\text { uma associação estatisticamente significativa entre o impacto } \\
\text { em qualidade de vida autopercebido e o grau de disfunção na } \\
\text { deglutição. }\end{array}$ \\
\hline $\begin{array}{l}\text { Prediction of aspiration in dysphagia } \\
\text { using logistic regression: oral intake } \\
\text { and self-evaluation12 }\end{array}$ & $\begin{array}{l}\text { Heijnen, B. J. } \\
\text { (2019) }\end{array}$ & $\begin{array}{l}\text {-Swallowing Quality of Life Questionnaire } \\
\text { (SWAL-QOL); } \\
\text {-Dysphagia Handicap Index (DHI). } \\
\text {-The Eating Assessment Tool } 10 \text { (EAT-10); }\end{array}$ & $\begin{array}{l}\text { Em pacientes com disfagia orofaríngea, os questionários auto- } \\
\text { reportados que avaliam statusfuncional de saúde equalidade de } \\
\text { vida relacionada à saúde podem ser usados num modelo } \\
\text { preditivo para avaliar o risco de aspiração, através de um } \\
\text { protocolo simples, não invasivo e custo-efetivo. O modelo } \\
\text { preditivo final apresentou AUC }=0.92 \text {, indicando boa } \\
\text { performance, mas não foi validado externamente. } \\
\text { Os pacientes eram classificados de acordo com a Escala } \\
\text { Funcional de Ingestão por Via Oral (do inglês, The Functional Oral } \\
\text { Intake Scale - FOIS). }\end{array}$ \\
\hline 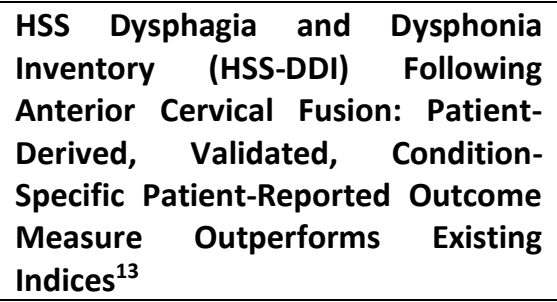 & $\begin{array}{l}\text { Hughes, A. P. } \\
\text { (2018) }\end{array}$ & $\begin{array}{l}\text { Special Surgery Dysphagia and Dysphonia } \\
\text { Inventory (HSS-DDI). Tradução livre - } \\
\text { Inventário de Disfonia e Disfagia em } \\
\text { Cirurgias }\end{array}$ & $\begin{array}{l}\text { O HSS-DDI aborda desfechos relacionados à disfonia e à disfagia } \\
\text { em pacientes submetidos à cirurgia de medula cervical com } \\
\text { abordagem anterior. Ele mostrou-se eficiente, válido e mais } \\
\text { efetivo nessa população - que apresenta um quadro dinâmico - } \\
\text { quando comparado a ferramentas existentes e disponíveis para } \\
\text { doenças crônicas. O HSS-DDI não foi validado externamente. }\end{array}$ \\
\hline
\end{tabular}

Continua... 


\section{Continuação}

\begin{tabular}{|c|c|c|c|}
\hline Artigo & $\begin{array}{l}\text { Autor principal } \\
\text { (Ano) }\end{array}$ & Questionário avaliado & Resultados \\
\hline $\begin{array}{l}\text { Quality-of-life scores compared to } \\
\text { objective measures of swallowing } \\
\text { after } \\
\text { chemoradiation }^{14} \quad \text { oropharyngeal }\end{array}$ & $\begin{array}{l}\text { Kendall, } \\
\text { Katherine A. } \\
\qquad(2014)\end{array}$ & $\begin{array}{l}\text {-MD Anderson Dysphagia Inventory } \\
\text { (MDADI) } \\
\text {-The University of Washington Swallowing } \\
\text { Quality of Life Questionnaire (UW-QOL). }\end{array}$ & $\begin{array}{l}\text { Não se estabeleceu correlação significativa entre as medidas } \\
\text { objetivas de distúrbios da deglutição (exame objetivo com } \\
\text { fluoroscopia da deglutição) e os escores coletados de qualidade } \\
\text { de vida. } \\
\text { A percepção do paciente sobre o impacto da disfagia na } \\
\text { qualidade de vida não se correlaciona com a sua função } \\
\text { fisiológica atual da deglutição. }\end{array}$ \\
\hline $\begin{array}{l}\text { The Dysphagia handicap index: } \\
\text { development and validation }{ }^{15}\end{array}$ & $\begin{array}{l}\text { Silbergleit, } \\
\text { Alice K. } \\
\text { (2012) }\end{array}$ & Dysphagia Handicap Index (DHI) & $\begin{array}{l}\text { O DHI é um questionário com } 25 \text { itens, auto reportado, aplicável } \\
\text { a indivíduos com disfagia de diferentes etiologias. A ferramenta } \\
\text { apresentou bons parâmetros de aplicabilidade e confiabilidade, } \\
\text { inclusive em indivíduos com menores níveis de alfabetização. }\end{array}$ \\
\hline $\begin{array}{l}\text { The Dysphagia Short Questionnaire, } \\
\text { an instrument for evaluation of } \\
\text { dysphagia: a validation study with } 12 \\
\text { months' follow-up after anterior } \\
\text { cervical spine surgery }{ }^{16}\end{array}$ & $\begin{array}{l}\text { Skeppholm, M. } \\
\text { (2012) }\end{array}$ & $\begin{array}{l}\text { - Dysphagia Short Questionnaire (DSQ) } \\
\text {-EQ-5D, questionário que avalia qualidade } \\
\text { de vida e status de saúde genérico. }\end{array}$ & $\begin{array}{l}\text { O DSQ teve uma correlação estatisticamente significativa ( } r= \\
0.59, \mathrm{P}<0.01 \text { ) com o MDADI, ferramenta conhecida e validada } \\
\text { para avaliar disfagia, permitindo concluir que o HSQ avalia } \\
\text { disfagia quantitativamente. Porém, o DSQ obteve fraca } \\
\text { correlação com o EQ-5D ( } r=-0.27, \mathrm{P}=0.05) \text {, com fraca correlação } \\
\text { na avaliação da qualidade de vida e status de saúde geral. }\end{array}$ \\
\hline $\begin{array}{l}\text { Defining Swallowing-Related Quality } \\
\text { of Life Profiles in Individuals with } \\
\text { Amyotrophic Lateral Sclerosis }^{17}\end{array}$ & $\begin{array}{l}\text { Tabor, L. } \\
\text { (2016) }\end{array}$ & $\begin{array}{l}\text {-Questionário The Swallowing Quality of } \\
\text { Life (SWAL-QOL); } \\
\text {-Escala Funcional Revisada de Pacientes } \\
\text { com Esclerose Lateral Amiotrófica (do } \\
\text { inglês, ALS Functional Rating Scale - } \\
\text { Revised-ALSFRS-R) }\end{array}$ & $\begin{array}{l}\text { Uma correlação positiva foi encontrada entre o SWAL-QOL e } \\
\text { ALSFRS-R ( } r=0.23, p=0.05) \text {. Maiores escores na escala ALSFRS- } \\
R \text { foram associados a maiores escores no questionário de } \\
\text { qualidade de vida relacionado à deglutição. Isso denota que } \\
\text { quanto maior o impacto funcional em pacientes com ELA, mais } \\
\text { comprometida é a sua qualidade de vida. Ademais, o SWAL-QOL } \\
\text { demonstrou associação negativa com a Escala de Penetração e } \\
\text { Aspiração. }\end{array}$ \\
\hline $\begin{array}{l}\text { Dysphagia and swallowing-related } \\
\text { quality of life in Friedreich ataxia18 }\end{array}$ & $\begin{array}{l}\text { Vogel, A. P. } \\
\text { (2014) }\end{array}$ & $\begin{array}{l}\text { Australian therapy outcome measures for } \\
\text { speech and swallowing (AusTOMS) }\end{array}$ & $\begin{array}{l}\text { Uma correlação significantemente positiva foi encontrada entre } \\
\text { a gravidade da deficiência, atividade, participação e sofrimento / } \\
\text { bem-estar no AusTOMS, sugerindo que a função de deglutição } \\
\text { diminui conforme reduções na qualidade de vida. }\end{array}$ \\
\hline
\end{tabular}

Continua... 


\section{Continuação}

\begin{tabular}{|l|c|l|l|}
\hline \multicolumn{1}{|c|}{ Artigo } & $\begin{array}{c}\text { Autor principal } \\
\text { (Ano) }\end{array}$ & Questionário avaliado & Resultados \\
\hline $\begin{array}{l}\text { Dysphagia-related quality of life in } \\
\text { oculopharyngeal } \begin{array}{c}\text { muscular } \\
\text { dystrophy: Psychometric properties } \\
\text { of the SWAL-QOL instrument19 }\end{array}\end{array}$ & $\begin{array}{c}\text { Youssof, S. } \\
\text { (2017) }\end{array}$ & $\begin{array}{l}\text { Swallowing Quality of Life instrument } \\
\text { (SWAL-QOL); }\end{array}$ & $\begin{array}{l}\text { A qualidade de vida relacionada à deglutição está reduzida em } \\
\text { pacientes com distrofia muscular oculofaríngea (do inglês, } \\
\text { limitações do SWAL-QOL, elaborado genericamente, uma } \\
\text { ferramenta específica que avalie qualidade de vida nos pacientes } \\
\text { com POMD é necessária. }\end{array}$ \\
\hline
\end{tabular}

A tabela acima contém a descrição qualitativa dos principais dados coletados de cada artigo incluído nessa revisão, evidencia ndo autor principal e ano de publicação, tipo de estudo, o questionário avaliado e uma descrição dos principais resultados encontrados no estudo. 
Análise de ferramentas utilizadas para avaliar o impacto da disfagia em qualidade de vida Bezerra MPRD, et al

\section{DISCUSSÃO}

O questionário Quality of Life in Swallowing Disorders (SWAL-QOL) baseia-se na medição quantitativa dos desfechos relatados, fornecidos pelos próprios pacientes. Esses dados são inseridos e avaliados em onze escalas diferentes, estruturadas por meio de uma pontuação cujo intervalo abrange os valores compreendidos entre " 0 " e " 100 ", nos quais os valores mais baixos representam pior qualidade de vida relacionada à deglutição. O seu desenvolvimento visa à utilização em pacientes com disfagia por diferentes etiologias, além de contribuir para o seguimento da alteração na qualidade de vida após uma intervenção terapêutica e exibir um impacto significativo na avaliação da saúde mental do grupo analisado.

Youssof et al. (2017) analisou o uso do SWAL-QOL em pacientes com Distrofia Muscular Óculo Faríngea (OPMD). Todas as escalas do SWAL-QOL demonstraram confiabilidade de consistência interna satisfatória, e a maioria demonstrou confiabilidade teste-reteste adequada. Apesar disso, existem limitações do uso da escala nessa população. O estudo pontua que pode haver alterações específicas da OPMD que não são abordadas pelo questionário, além da presença do efeito piso/teto em 7 de 10 escalas, levando a diminuição da capacidade discriminativa dessas.

Nesse contexto, o estudo desenvolvido por Cordier et al. (2018), que analisa o SWAL-QOL em população de 507 indivíduos com risco de disfagia orofaríngea utilizando enquanto método a Teoria de Resposta ao Item (TRI) - constata que a ferramenta necessita de reformulações. Tal conclusão decorre da presença, no questionário, de itens inadequados ou redundantes, além de potenciais lacunas nas descrições de algumas categorias que resultam na perda de precisão quanto ao objeto que está sendo abordado, levando o paciente a apresentar respostas duvidosas. 0 estudo em questão não aprova o uso do questionário.

O estudo realizado por Heijnen et al. (2019) utiliza o SWAL-QOL e o Dysphagia Handicap Index (DHI), juntamente com outros questionários, para desenvolver uma ferramenta de avaliação não invasiva acerca da aspiração em pacientes com disfagia orofaríngea. O modelo obteve boa acurácia pelo estudo, mas não foi validado externamente.

Florie et al. (2016) realizou uma coorte de 63 pacientes tratados para câncer de cabeça e pescoço, com o objetivo de determinar se existia associação entre a função da deglutição, avaliada pela Vídeo-endoscopia da deglutição (VED) e os resultados do questionário o "MD Anderson Dysphagia Inventory" (MDADI). A ferramenta se mostrou eficiente para avaliar o impacto da doença na qualidade de vida dos indivíduos, mas não pode ser correlacionada com a gravidade da condição clínica apresentada.

Hughes et al. (2018) desenvolveram o questionário Hospital for Special Surgery Dysphagia and Dysphonia Inventory (HSS-DDI) para avaliar queixas de disfagia e disfonia 
Análise de ferramentas utilizadas para avaliar o impacto da disfagia em qualidade de vida Bezerra MPRD, et al

em pacientes pós cirurgia da coluna cervical. Trata-se de um estudo multifásico para detecção das principais queixas e elaboração de questionário com itens sensíveis no checklist. No estudo, houve seleção dos pacientes que fizeram cirurgias mais extensas e tinham quadros mais graves, ou seja, seriam de maior risco para disfagia/disfonia. Isso poderia superestimar a sensibilidade da ferramenta.

O Dysphagia Handicap Index (DHI) considera três subescalas de avaliação: emocional, funcional e uma avaliação do impacto físico. É um questionário auto reportado com 25 itens, desenvolvido e validado para avaliar disfagia de múltiplas etiologias, incluindo pacientes com doenças neurodegenerativas, DRGE, alterações estruturais e pacientes oncológicos. O objetivo da criação desse questionário, validado por Silbergleit et al. (2012) em mais de 200 pacientes, foi desenvolver um questionário mais prático e simples de ser entendido pelos pacientes. $\mathrm{O} \mathrm{DH}$ obteve relevância estatística em diferenciar indivíduos com disfagia dos controles, com uma alta validade interna, além de boa capacidade discriminatória nos graus de disfagia.

Em um estudo por Tabor et al. (2016) em pacientes com Esclerose Lateral Amiotrófica (ELA), foi observado uma correlação estatisticamente significativa entre a pontuação do SWAL-QOL e a progressão da doença, medida pela escala Global disease progression (ALSFRS-R score). Isso denota que quanto mais avançado o estágio da doença, maior a percepção do paciente de impacto na qualidade de vida.

Skeppholm (2012) propôs com seu estudo analisar a validade e confiabilidade do Dysphagia Short Questionnaire (DSQ), comparando-o com o MDADI - por esse último se tratar de um sistema de pontuação já validado, de modo que se verificou ser satisfatória a reprodutibilidade e facilidade de uso do DSQ por ser semelhante ao MDADI.

Já o estudo desenvolvido por Vogel et al. (2014) utiliza o questionário Royal Brisbane Hospital Outcome Measure for Swallowing (RBHOMS) para avaliação da disfagia na ataxia de Friedreich. O método se baseia em uma escala de dez pontos, que mede a ingestão oral, a função de deglutição e a dependência relativa da alimentação por sonda enteral do paciente. Ao ser aplicado, resultou em um estudo que demonstra o quão frequentes são os transtornos da deglutição nessa patologia. Ainda, verificou-se que a associação do questionário com outras formas de avaliação dos pacientes foi substancial para desenvolver estratégias compensatórias ou modificação da dieta tanto na ingestão de alimentos quanto nos líquidos -, fundamental para um tratamento eficaz.

Um achado interessante desses estudos é que o impacto em qualidade de vida não apresenta correlação estatisticamente significativa com a gravidade do problema, isto é, quanto mais grave a disfunção na deglutição avaliada por medidas instrumentais - como a VED -, não necessariamente pior é a percepção do paciente sobre o comprometimento psicossocial respondido em questionários com esse propósito. 
Análise de ferramentas utilizadas para avaliar o impacto da disfagia em qualidade de vida Bezerra MPRD, et al

Como demonstrou Florie et al. (2016) em pacientes tratados para câncer de cabeça e pescoço, os achados na VED não obtiveram associação linear com a pontuação nos escores de qualidade de vida. Assim como Kendall et al. (2014) revelou não haver correlação da avaliação da disfagia pelos escores SWAL-QOL ou MDADI e medidas objetivas da função da deglutição aferidas pelo Modified barium swallow studies em pacientes com câncer orofaríngeo após quimiorradioterapia. A reflexão que isso levanta é que mesmo disfunções leves podem acarretar grande impacto psíquico e social para certos pacientes, enquanto outros podem apresentar maior tolerabilidade ao problema. A percepção subjetiva da doença depende do status social do indivíduo, grau de escolaridade e seu limiar individual para o sofrimento.

Dentre as limitações encontradas na elaboração desta revisão, a estratégia de busca e os critérios usados para seleção dos artigos compilaram evidências bastante heterogêneas, tanto pelo seu desenho de estudo e metodologia, quanto pela pergunta de pesquisa a ser respondida. Ademais, a análise e a percepção dos autores sobre os estudos incluídos são de uma divergência metodológica e conceitual significativa entre as evidências. Um outro ponto importante a ser mencionado é acerca do viés de publicação, cuja análise subjetiva revelou uma tendência à inclusão de estudos com resultados positivos.

\section{CONCLUSÃO}

Diversas ferramentas são utilizadas para avaliar a qualidade de vida relacionada à saúde em pacientes com disfagia orofaríngea, mas, em sua maioria, esses instrumentos não estão devidamente validados ou seu uso é sustentado por estudos de baixa qualidade metodológica e são pouco confiáveis. Um outro problema identificado é a existência de múltiplas ferramentas distintas e a ausência de um instrumento padronizado para esta condição clínica.

O SWAL-QOL é a ferramenta mais estudada, traduzida para mais línguas e cujas análises psicométricas mostraram melhores resultados, mas novos estudos vêm questionando a qualidade desse instrumento.

Assim, reforçamos a importância de estudar objetivamente a correlação entre qualidade de vida de disfagia orofaríngea, mas reiteramos a necessidade de melhores estudos para desenvolvimento de ferramentas com melhor acurácia.

\section{REFERÊNCIAS}

1. Rommel N, Hamdy S. Oropharyngeal dysphagia: manifestations and diagnosis. Nat Rev Gastroenterol Hepatol. 2016;13(1):49-59.

2. Eslick GD, Talley NJ. Dysphagia: epidemiology, risk factors and impact on quality of life--a population-based study. Aliment Pharmacol Ther. 2008;27(10):971-9. 
Análise de ferramentas utilizadas para avaliar o impacto da disfagia em qualidade de vida Bezerra MPRD, et al

3. Carrion S, Cabr M, Monteis R, Roca M, Palomera E, Serra-Prat M, et al. Oropharyngeal dysphagia is a prevalent risk factor for malnutrition in a cohort of older patients admitted with an acute disease to a general hospital. Clin Nutr. 2015;34(3):436-42.

4. Almirall J, Rofes L, Serra-Prat M, Icart R, Palomera E, Arreola V, et al. Oropharyngeal dysphagia is a risk factor for community-acquired pneumonia in the elderly. Eur Respir J. 2013;41(4):923-8.

5. Ekberg O, Hamdy S, Woisard V, Wuttge-Hannig A, Ortega P. Social and psychological burden of dysphagia: its impact on diagnosis and treatment. Dysphagia. 2002;17(2):139-46.

6. Bedaque $\mathrm{H}$, Lidiane Maria de Brito Macedo Ferreira, Fernandes KM, Godoy CM de A, Magalhães Junior HV. OROPHARYNGEAL DYSPHAGIA: AN ASSOCIATION BETWEEN DYSPHAGIA LEVEL, SYMPTOMS AND COMORBIDITY. J Surg Cl Res [Internet]. 2020May22 [cited 2021Apr.12];11(1):39-5. Available from: https://periodicos.ufrn.br/jscr/article/view/20955

7. Timmerman AA, Speyer R, Heijnen BJ. Psychometric Characteristics of HealthRelated Quality-of-Life Questionnaires in Oropharyngeal Dysphagia. Dysphagia. 2014; 29, 183-198.

8. Chen L. Overview of clinical prediction models. Ann Transl Med. 82020. p. 71.

9. Steyerberg EW, Van Calster B, Pencina MJ. [Performance measures for prediction models and markers: evaluation of predictions and classifications]. Rev Esp Cardiol. 2011;64(9):788-94.

10. Cordier R, Speyer R, Schindler A, Michou E, Heijnen BJ, Baijens L, et al. Using Rasch Analysis to Evaluate the Reliability and Validity of the Swallowing Quality of Life Questionnaire: An Item Response Theory Approach. Dysphagia. 2018;33(4):441-56.

11. Florie M, Baijens L, Kremer B, Kross K, Lacko M, Verhees F, et al. Relationship between swallow-specific quality of life and fiber-optic endoscopic evaluation of swallowing findings in patients with head and neck cancer. Head Neck. 2016;38 Suppl 1:E1848-56.

12. Heijnen BJ, Bohringer S, Speyer R. Prediction of aspiration in dysphagia using logistic regression: oral intake and self-evaluation. Eur Arch Otorhinolaryngol. 2020;277(1):197-205.

13. Hughes AP, Salzmann SN, Aguwa OK, Miller CO, Duculan R, Shue J, et al. HSS Dysphagia and Dysphonia Inventory (HSS-DDI) Following Anterior Cervical Fusion: Patient-Derived, Validated, Condition-Specific Patient-Reported Outcome Measure Outperforms Existing Indices. J Bone Joint Surg Am. 2018;100(10):e66.

14. Kendall KA, Kosek SR, Tanner K. Quality-of-life scores compared to objective measures of swallowing after oropharyngeal chemoradiation. The Laryngoscope. 2014;124(3):682-7.

15. Silbergleit AK, Schultz L, Jacobson BH, Beardsley T, Johnson AF. The Dysphagia handicap index: development and validation. Dysphagia. 2012;27(1):46-52. 
Análise de ferramentas utilizadas para avaliar o impacto da disfagia em qualidade de vida Bezerra MPRD, et al

16. Skeppholm M, Ingebro C, Engstrom T, Olerud C. The Dysphagia Short Questionnaire: an instrument for evaluation of dysphagia: a validation study with 12 months' follow-up after anterior cervical spine surgery. Spine (Phila Pa 1976). 2012;37(11):996-1002.

17. Tabor L, Gaziano J, Watts S, Robison R, Plowman EK. Defining Swallowing-Related Quality of Life Profiles in Individuals with Amyotrophic Lateral Sclerosis. Dysphagia. 2016;31(3):376-82.

18. Vogel AP, Brown SE, Folker JE, Corben LA, Delatycki MB. Dysphagia and swallowingrelated quality of life in Friedreich ataxia. J Neurol. 2014;261(2):392-9.

19. Youssof S, Romero-Clark C, Warner T, Plowman E. Dysphagia-related quality of life in oculopharyngeal muscular dystrophy: Psychometric properties of the SWAL-QOL instrument. Muscle Nerve. 2017;56(1):28-35. 